\title{
Prevalence of Corynebacterium ulcerans in dogs in Osaka, Japan
}

\author{
Chihiro Katsukawa, ${ }^{1}$ Takako Komiya, ${ }^{2}$ Hiroaki Yamagishi, ${ }^{3}$ Atsushi Ishii, ${ }^{3}$ \\ Shunji Nishino, ${ }^{3}$ Shinya Nagahama, ${ }^{4}$ Masaaki Iwaki, ${ }^{2}$ Akihiko Yamamoto ${ }^{2}$ \\ and Motohide Takahashi ${ }^{2}$ \\ ${ }^{1}$ Department of Infectious Diseases, Osaka Prefectural Institute of Public Health, Osaka, Japan \\ ${ }^{2}$ Department of Bacteriology II, National Institute of Infectious Diseases, Tokyo, Japan \\ ${ }^{3}$ Osaka Prefectural Dog Management Office, Osaka, Japan \\ ${ }^{4}$ Animal Protection and Livestock Division, Department of Environment, Agriculture, \\ Forestry and Fisheries, Osaka Prefectural Government, Osaka, Japan
}

Correspondence

Chihiro Katsukawa

katukawa@iph.pref.osaka.jp

Masaaki Iwaki

miwaki@nih.go.jp

Received 16 June 2011

Accepted 14 September 2011

\section{INTRODUCTION}

Some strains of Corynebacterium ulcerans produce diphtheria toxin and may cause human infection with diphtheria-like symptoms. In Japan, eight cases of infection by toxigenic $C$. ulcerans in humans were reported between 2001 and 2011 (Asakura et al., 2006; Hagiwara et al., 2006; Hatanaka et al., 2003, 2011; Komiya et al., 2010; Noguchi et al., 2009; Nureki et al., 2007; Yoshimura et al., 2010). C. ulcerans causes purulent inflammatory diseases such as mastitis, lymphadenitis, dermatitis and respiratory infections in various domestic and wild animals. Human infections are caused by ingestion of untreated milk (Bostock et al., 1984) or by close contact with a variety of animals (De Zoysa et al., 2005; Hatanaka et al., 2011; Noguchi et al., 2009; Schuhegger et al., 2009; Tiwari et al., 2008; Yoshimura et al., 2010). Dogs are of pronounced importance because they are sometimes linked to serious human cases (Hogg et al., 2009; Lartigue et al., 2005).

Abbreviations: CLDM, clindamycin; CLSI, Clinical and Laboratory Standards Institute; LVFX, levofloxacin.

The GenBank/EMBL/DDBJ accession numbers for the tox sequences of C. ulcerans Dog0803, Dog0804 and Dog0811 and C. diphtheriae ATCC 11049, ATCC 11051, RIMD 0343044 and CD1994-1 are AB602353, AB602354, AB602355, AB602356, AB602357, $\mathrm{AB} 602358$ and $\mathrm{AB} 602359$, respectively.

Supplementary tables are available with the online version of this paper.
To determine whether domestic dogs serve as a reservoir for human C. ulcerans infection, we carried out a series of initial screenings for C. ulcerans in 65 healthy dogs who were, for various reasons, in the custody of the Osaka Prefectural Government from December 2006 to September 2007. Toxigenic C. ulcerans was isolated from one dog (Katsukawa et al., 2009). C. ulcerans was not found in any of the 218 family dogs living in the area where the infected dog originated (Takahashi, 2009).

In this study, we report the results of our continuation of the screening for C. ulcerans in the dogs in custody. In order to compensate for any seasonal effects on the screening, the screening period was extended to cover 13 months from November 2007 to December 2008. Upon examination of 583 dogs, 45 C. ulcerans isolates were obtained from 44 dogs. The characteristics of these isolates are described here.

\section{METHODS}

Dogs and sample collection. Between 27 November 2007 and 26 December 2008, 944 adult dogs were under the care of the Osaka Prefectural Government. Due to a tight schedule for euthanizing the dogs, throat swabs were collected from only 583 of the 944 dogs. The test population included 363 male and 220 female (401 mongrels and 182 purebreds) dogs. These dogs were either abandoned or had escaped from their guardians. Throughout the screening period, 
sample collection was usually carried out twice a week, on Tuesdays and Fridays. Throat swabs were collected immediately after euthanasia and stored in preservation medium (SEEDSWAB $\gamma 3$ 'Eiken'; Eiken Chemical) at $4{ }^{\circ} \mathrm{C}$.

Bacterial isolation and identification. Bacterial strains used in this study are listed in Table 1. For the isolation of bacteria, culturing was started on the day of sample collection by inoculating the swabs on sheep blood agar and selective medium (charcoaltellurite blood agar), and incubating them at $35{ }^{\circ} \mathrm{C}$ (Katsukawa et al., 2009). Charcoal-tellurite blood agar contains heart infusion agar, $0.03 \%(\mathrm{w} / \mathrm{v})$ potassium tellurite, $10 \%(\mathrm{v} / \mathrm{v})$ sheep blood and $0.05 \%$ (w/v) activated charcoal (Katsukawa et al., 2009). Colonies appearing after 18-24 h on sheep blood agar and after 24, 30 and $48 \mathrm{~h}$ on charcoal-tellurite blood agar were transferred to dextrosesucrose-starch agar medium (Jacherts, 1956) to investigate glucose and sucrose fermentation. Isolates positive for glucose but negative for sucrose fermentation were then characterized by Gram staining and by the catalase and urease tests. Thus, all Gram-positive organisms that tested positive for catalase and urease production were suspected to be C. ulcerans and further analysed using an API Coryne (bioMérieux) kit, followed by the determination of partial RNA polymerase $\beta$-subunit $(r p o B)$ sequences (Khamis et al., 2004).

Toxigenicity testing and nucleotide sequencing of the tox gene. Toxigenicity testing was performed by modified Elek test (Engler et al., 1997; Katsukawa et al., 2009; Reinhardt et al., 1998), Vero cell cytotoxicity and neutralization tests (Katsukawa et al., 2009;

Table 1. Bacterial strains and isolates used in this study

Strains used for comparison studies are listed here.

\begin{tabular}{|c|c|c|}
\hline Isolate/strain & Source & Reference \\
\hline \multicolumn{3}{|l|}{$\begin{array}{l}\text { Corynebacterium } \\
\text { ulcerans }\end{array}$} \\
\hline 0102 & Human & $\begin{array}{l}\text { Hatanaka et al. (2003); } \\
\text { Komiya et al. (2010) }\end{array}$ \\
\hline 0211 & Human & Komiya et al. (2010) \\
\hline 0509 & Human & Asakura et al. (2006) \\
\hline 0510 & Human & Nureki et al. (2007) \\
\hline 0607 & Human & Hagiwara et al. (2006) \\
\hline 0902 & Human & Noguchi et al. (2009) \\
\hline Dog0708 & Dog & Katsukawa et al. (2009) \\
\hline $\operatorname{Dog} 0803$ & Dog & This study \\
\hline Dog0804 & Dog & This study \\
\hline $\operatorname{Dog} 0807$ & Dog & This study \\
\hline Dog0809-1 & Dog & This study \\
\hline Dog0809-2 & Dog & This study \\
\hline Dog0809-3 & Dog & This study \\
\hline Dog0811 & Dog & This study \\
\hline \multicolumn{3}{|l|}{$\begin{array}{l}\text { Corynebacterium } \\
\text { diphtheriae }\end{array}$} \\
\hline ATCC 11049 & Human & ATCC \\
\hline ATCC 11051 & Human & ATCC \\
\hline RIMD 0343044 & Human & RIMD $^{*}$ \\
\hline CD1994-1 & Human & Thailand (this study) \\
\hline
\end{tabular}

${ }^{\star}$ Culture Collection of the Research Institute for Microbial Diseases, Osaka University, Japan.
Miyamura et al., 1974) and PCR for the diphtheria toxin (tox) gene. Primers used for PCR are listed in Supplementary Table S1 in JMM Online. Primers Tox 1 and Tox 2 (Nakao \& Popovic, 1997), corresponding to the A subunit of the toxin, were first used for detection of the tox gene. In isolates negative for the primer pair, further PCR analyses using primers Dipht 6F and Dipht 6R (Nakao et al., 1996) for the B subunit and toxFw and toxRv for the entire tox gene (Seto et al., 2008) were carried out to confirm the absence of the gene. The nucleotide sequence of the tox gene was determined by amplifying the entire gene fragment with primers toxFw and toxRv (Seto et al., 2008) (Supplementary Table S1) and then by sequencing the fragment with primers placed at appropriate intervals. A phylogenetic tree of tox nucleotide sequences was constructed using the neighbour-joining method (Saitou \& Nei, 1987).

PFGE. PFGE was performed on all 45 isolates as well as on the $C$. ulcerans strains previously isolated from dogs and humans in Japan. PFGE of Sfil-digested genomic bacterial DNA (De Zoysa et al., 1995) was performed using $1.5 \%(\mathrm{w} / \mathrm{v})$ agarose gels and $0.5 \times$ Tris/borate EDTA buffer at $14{ }^{\circ} \mathrm{C}, 6 \mathrm{~V} \mathrm{~cm}{ }^{-1}, 5-20 \mathrm{~s}$ pulse for $18 \mathrm{~h}$, followed by 1-5 s pulse for $14 \mathrm{~h}$, using a CHEF-DR II system (Bio-Rad). PFGE patterns were analysed with the Diversity Database software by the UPGMA algorithm.

Antibiotic susceptibility. Antibiotic susceptibility tests were performed by the broth microdilution method using a Dry Plate DP24 (Eiken Chemical). We tested the following antibiotics: benzylpenicillin, ampicillin, cefazolin, cefotiam, cefotaxime, cefaclor, cefditoren, flomoxef, imipenem, meropenem, erythromycin, clindamycin (CLDM), minocycline, vancomycin, levofloxacin (LVFX) and sulfamethoxazole-trimethoprim. Sensitivities were assessed according to the Clinical and Laboratory Standards Institute's (CLSI) standard (M45-A) for Corynebacterium species. To assign sensitivities to the eight drugs for which CLSI standard values are not available, standards for similar drugs were considered (sensitivity to ampicillin tested using benzylpenicillin sensitivity standard; cefazolin, cefotiam, cefaclor, cefditoren and flomoxef tested using cefotaxime standard; minocycline tested using tetracycline standard; and LVFX tested using ciprofloxacin sensitivity standard).

\section{RESULTS}

\section{Isolation of C. ulcerans and toxigenicity}

Fig. 1 displays the number of isolates obtained over the course of the study. Supplementary Table S2 also summarizes the study population with respect to gender and seasonality of $C$. ulcerans isolation. A more comprehensive summary of the C. ulcerans isolates is shown in Table 2.

The first isolate was detected in March 2008, followed by the isolation of indistinguishable isolates from three other dogs that had the same guardian. These four isolates were classified as group 1. Screening continued intermittently, with 27 C. ulcerans-positive dogs identified between April and the beginning of June (group 2), six in July (group 3) and none until September. Seven isolates were obtained from six dogs in September and October (group 4, subdivided into three subgroups, $4-1$ to $4-3$, by toxigenicity and PFGE patterns) and one isolate from one dog in November (group 5). In summary, 45 C. ulcerans isolates (42 toxigenic and three non-toxigenic) were obtained from 44 dogs. From one of these dogs, toxigenic and 


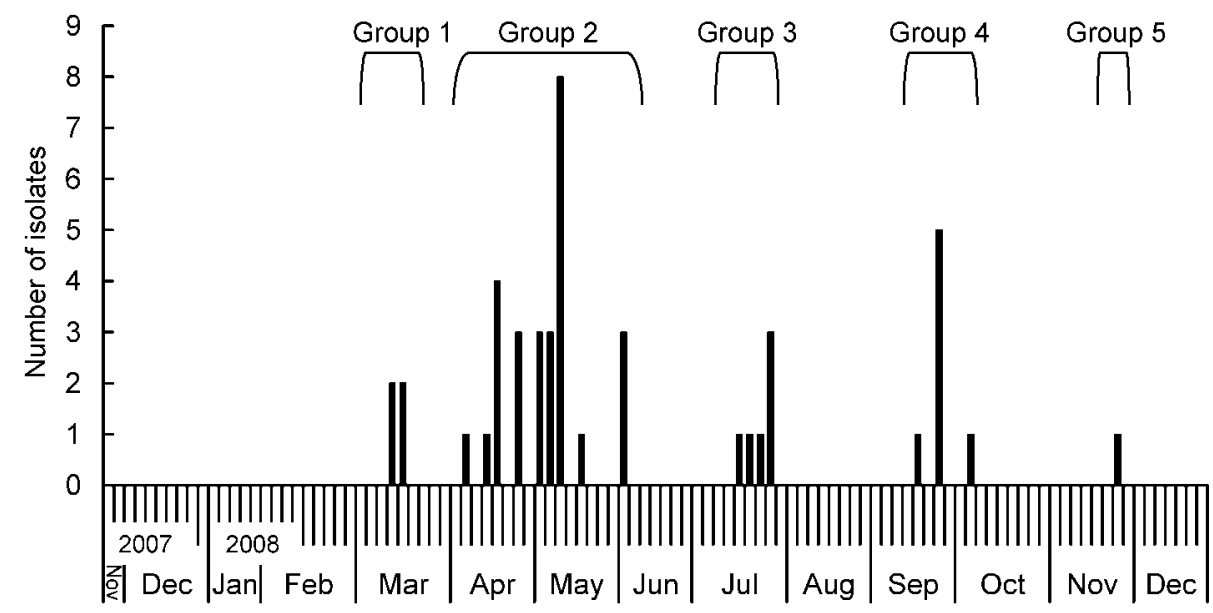

Fig. 1. Chronological representation of C. ulcerans isolation. Samples were collected as described in the text. Each sampling period is expressed as a small cell between the horizontal axis and month indications. Bars represent the number of isolates obtained during each sampling period.

non-toxigenic isolates were isolated simultaneously (Table 2 , nos 39 and 40). For each of the other dogs, one isolate was obtained per dog (41 toxigenic and two non-toxigenic). None of the 44 dogs from which C. ulcerans was isolated were apparently symptomatic: nasal discharge, pharyngitis and dermatitis were not observed. There was no correlation between detection rates and the sex of the animals.

Each of the 45 isolates were obtained from the primary culture growing on charcoal-tellurite blood agar plates. Isolation from primary cultures on blood agar was not successful: only three isolates were obtained, mainly due to masking by the growth of other bacterial species present on the swab. The three isolates were later proven to be identical to the isolates obtained from the charcoal-tellurite blood agar plates of the same specimens.

All strains tested positive for catalase and urease production and glucose fermentation and negative for sucrose fermentation. They shared the same API code $(0111326, \%$ id 99.7, $T=1.0$ ). Forty-two isolates were toxigenic, as demonstrated by positive results of PCR for the tox gene (A subunit), modified Elek test, and Vero cell cytotoxicity and neutralization tests. Three isolates were negative for all of these tests and were thus considered to be non-toxigenic. Detailed characteristics of these isolates are summarized in Table 2.

\section{PFGE analysis}

All 45 isolates and the previous dog and human isolates were compared using PFGE. The isolates were classified into four types (A2, B, C, D) by PFGE (Table 2 and Fig. 2). PFGE classifications of isolates were as follows: PFGE type $\mathrm{C}$ included group 1 isolates (four toxigenic strains isolated in March); type A2 included groups 2, 3, 4-1 and 4-2 (37 toxigenic strains isolated from April to October and two of three non-toxigenic strains isolated in September); type B included group 4-3 (one non-toxigenic strain isolated in September); and type D included group 5 (one toxigenic strain isolated in November). As shown in Fig. 2, subtype A2, together with two other subtypes classified according to slight differences in PFGE patterns, composed type A. Previously isolated human strains have been classified with respect to PFGE type as follows: PFGE type A1, human isolates 0102 and 0210 (Komiya et al., 2010) that were closely related to type A2, including 0509 (Asakura et al., 2006) and 0902 (Noguchi et al., 2009), and type A3 including 0607 (Hagiwara et al., 2006). Another human isolate, 0510 (Nureki et al., 2007), was classified in a comparatively distant type $\mathrm{D}$.

\section{Analysis of the diphtheria toxin (tox) gene}

The complete tox gene sequence (1683 bp) was determined for 42 toxigenic isolates for which toxigenicity was confirmed. Their sequences were divided into three types as shown in Table 2 and Fig. 3. The first type (designated tox0803; GenBank accession no. AB602353) included four isolates of group 1 including Dog0803; the second type (tox0804; GenBank accession no. AB602354) included 37 toxigenic isolates of groups 2, 3 and 4-1, obtained from April through October, including Dog0804; and the last type (tox0811; GenBank accession no. AB602355) included only one isolate, Dog0811 (group 5), obtained in November. These sequences were also compared to the previously published tox gene sequences of $C$. ulcerans human strains 0102, 0510, A6361, A2911, X959 and KL126 (Komiya et al., 2010; Schuhegger et al., 2009; Seto et al., 2008; Sing et al., 2003, 2005; Wellinghausen et al., 2002) (Table 3, Fig. 3). More than $98 \%$ similarity was shown between any two of the C. ulcerans isolates and strains. However, the sequences showed reduced similarity ( $\sim 95 \%)$ to the reported tox gene 
Table 2. Characteristics of the isolates

\begin{tabular}{|c|c|c|c|c|c|c|c|c|c|c|c|c|c|}
\hline \multirow[t]{2}{*}{ No. } & \multirow[t]{2}{*}{ Group } & \multirow{2}{*}{$\begin{array}{l}\text { Designation as } \\
\text { the isolate } \\
\text { representing } \\
\text { the group }\end{array}$} & \multirow[t]{2}{*}{$\begin{array}{c}\text { Date of } \\
\text { isolation }^{*}\end{array}$} & \multirow[t]{2}{*}{$\operatorname{Sex} \dagger$} & \multicolumn{2}{|c|}{$\begin{array}{c}\text { Isolation } \\
\text { on } \neq:\end{array}$} & \multirow{2}{*}{$\begin{array}{c}\text { API } \\
\text { Coryne } \\
\text { code }\end{array}$} & \multirow[t]{2}{*}{$\begin{array}{r}\text { tox } \\
\text { PCR }\end{array}$} & \multirow[t]{2}{*}{ Elek } & \multirow[t]{2}{*}{$\begin{array}{c}\text { Vero cell } \\
\text { cytotoxicity }\end{array}$} & \multirow[t]{2}{*}{$\begin{array}{l}\text { PFGE } \\
\text { type }\end{array}$} & \multirow{2}{*}{$\begin{array}{c}\text { tox } \\
\text { gene } \\
\text { type }\end{array}$} & \multirow[t]{2}{*}{$\begin{array}{c}\text { Drug } \\
\text { susceptibility } \$\end{array}$} \\
\hline & & & & & BA & $\mathrm{C}$ & & & & & & & \\
\hline 1 & 1 & Dog0803 & $3 / 11$ & $\mathrm{~F}$ & - & + & 0111326 & + & + & + & $\mathrm{C}$ & tox0803 & CLDM: R \\
\hline 4 & 1 & & $3 / 14$ & $\mathrm{~F}$ & - & + & 0111326 & + & + & + & $\mathrm{C}$ & tox0803 & CLDM: R \\
\hline 5 & 2 & Dog0804 & $4 / 4$ & M & - & + & 0111326 & + & + & + & $\mathrm{A} 2$ & tox0804 & CLDM: R \\
\hline 6 & 2 & & $4 / 11$ & $\mathrm{M}$ & - & + & 0111326 & + & + & + & $\mathrm{A} 2$ & tox0804 & CLDM: R \\
\hline 7 & 2 & & $4 / 15$ & $\mathrm{~F}$ & - & + & 0111326 & + & + & + & $\mathrm{A} 2$ & tox0804 & CLDM: R \\
\hline 8 & 2 & & $4 / 15$ & $\mathrm{~F}$ & - & + & 0111326 & + & + & + & A2 & tox0804 & CLDM: R \\
\hline 9 & 2 & & $4 / 15$ & M & - & + & 0111326 & + & + & + & $\mathrm{A} 2$ & tox0804 & CLDM: R \\
\hline 13 & 2 & & $4 / 22$ & $\mathrm{M}$ & - & + & 0111326 & + & + & + & $\mathrm{A} 2$ & tox0804 & CLDM: R \\
\hline 14 & 2 & & $5 / 2$ & $\mathrm{~F}$ & - & + & 0111326 & + & + & + & $\mathrm{A} 2$ & tox0804 & CLDM: R \\
\hline 15 & 2 & & $5 / 2$ & $\mathrm{M}$ & - & + & 0111326 & + & + & + & A2 & tox0804 & CLDM: R \\
\hline 16 & 2 & & $5 / 2$ & $\mathrm{M}$ & - & + & 0111326 & + & + & + & A2 & tox0804 & CLDM: R \\
\hline 17 & 2 & & $5 / 9$ & $\mathrm{M}$ & - & + & 0111326 & + & + & + & A2 & tox0804 & CLDM: $\mathrm{R}$ \\
\hline 18 & 2 & & $5 / 9$ & M & - & + & 0111326 & + & + & + & $\mathrm{A} 2$ & tox0804 & CLDM: R \\
\hline 19 & 2 & & $5 / 9$ & M & - & + & 0111326 & + & + & + & A2 & tox0804 & CLDM: $\mathrm{R}$ \\
\hline 20 & 2 & & $5 / 13$ & $\mathrm{~F}$ & - & + & 0111326 & + & + & + & $\mathrm{A} 2$ & tox0804 & CLDM: R \\
\hline 21 & 2 & & $5 / 13$ & $\mathrm{~F}$ & - & + & 0111326 & + & + & + & A2 & tox0804 & CLDM: R \\
\hline 22 & 2 & & $5 / 13$ & M & - & + & 0111326 & + & + & + & $\mathrm{A} 2$ & tox0804 & CLDM: R \\
\hline 23 & 2 & & $5 / 13$ & M & - & + & 0111326 & + & + & + & $\mathrm{A} 2$ & tox0804 & CLDM: R \\
\hline 32 & 3 & Dog0807 & $7 / 15$ & M & - & + & 0111326 & + & + & + & $\mathrm{A} 2$ & tox0804 & CLDM: I, LVFX: I \\
\hline 33 & 3 & & $7 / 18$ & M & - & + & 0111326 & + & + & + & $\mathrm{A} 2$ & tox0804 & CLDM: I, LVFX: I \\
\hline 34 & 3 & & $7 / 22$ & $\mathrm{~F}$ & - & + & 0111326 & + & + & + & $\mathrm{A} 2$ & tox0804 & CLDM: I, LVFX: I \\
\hline 35 & 3 & & $7 / 25$ & $\mathrm{~F}$ & - & + & 0111326 & + & + & + & A2 & tox0804 & CLDM: I, LVFX: I \\
\hline 36 & 3 & & $7 / 25$ & $\mathrm{~F}$ & - & + & 0111326 & + & + & + & A2 & tox0804 & CLDM: I, LVFX: I \\
\hline 37 & 3 & & $7 / 25$ & M & - & + & 0111326 & + & + & + & $\mathrm{A} 2$ & tox0804 & CLDM: I, LVFX: I \\
\hline 38 & $4-1$ & Dog0809-1 & $9 / 16$ & M & + & + & 0111326 & + & + & + & $\mathrm{A} 2$ & tox0804 & CLDM: I \\
\hline $39 \|$ & $4-1$ & & $9 / 26$ & M & - & + & 0111326 & + & + & + & $\mathrm{A} 2$ & tox0804 & CLDM: I \\
\hline $40 \|$ & $4-2$ & Dog0809-2 & $9 / 26$ & M & - & + & 0111326 & - & - & - & $\mathrm{A} 2$ & - & CLDM: I \\
\hline 41 & $4-3$ & Dog0809-3 & $9 / 26$ & M & - & + & 0111326 & - & - & - & B & - & CLDM: I \\
\hline 42 & $4-2$ & & $9 / 26$ & M & - & + & 0111326 & - & - & - & $\mathrm{A} 2$ & - & CLDM: I \\
\hline 43 & $4-1$ & & $9 / 26$ & M & + & + & 0111326 & + & + & + & $\mathrm{A} 2$ & tox0804 & CLDM: I \\
\hline 44 & $4-1$ & & $10 / 7$ & M & - & + & 0111326 & + & + & + & $\mathrm{A} 2$ & tox0804 & CLDM: I \\
\hline 45 & 5 & Dog0811 & $11 / 25$ & M & + & + & 0111326 & + & + & + & $\mathrm{D}$ & tox0811 & CLDM: R \\
\hline
\end{tabular}

${ }^{\star}$ Month/day in 2008.

$\dagger \mathrm{M}$, Male; F, female.

$\ddagger \mathrm{BA}$, Blood agar; $\mathrm{C}$, charcoal-tellurite blood agar.

$\S$ CLDM, Clindamycin; LVFX, levofloxacin; R, resistant; I, intermediate.

IIIsolated from the same dog. 


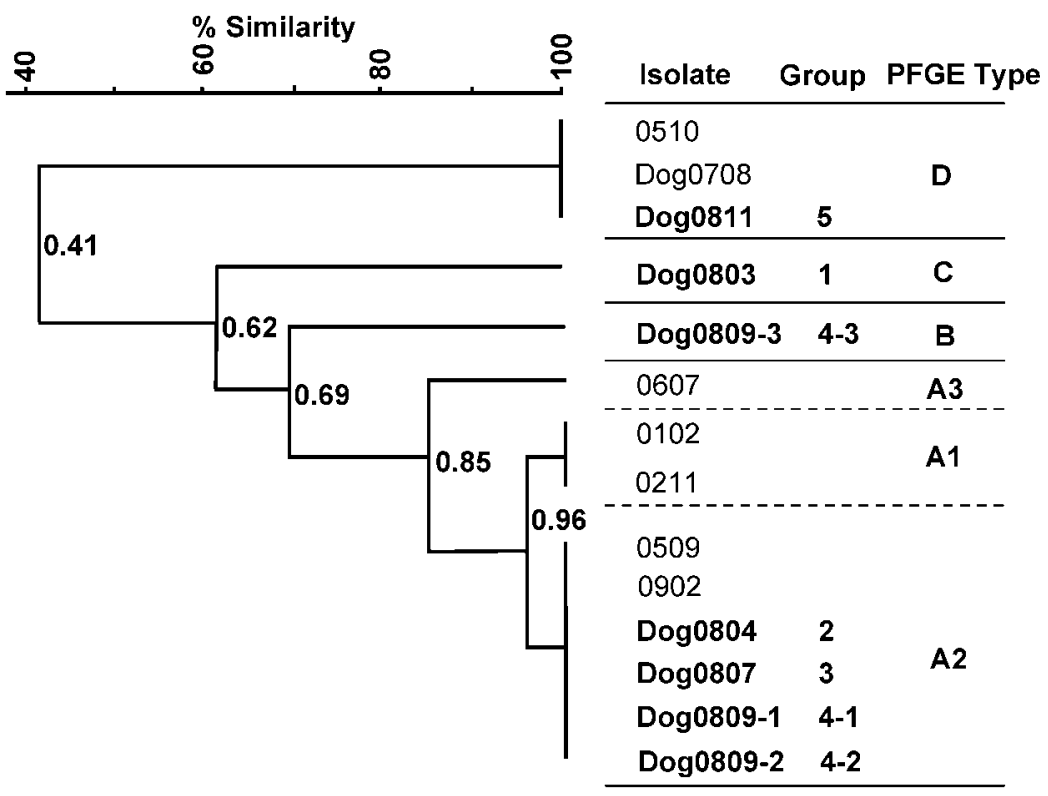

Fig. 2. Phylogenetic analysis of C. ulcerans isolates. C. ulcerans dog isolates obtained in this study (indicated in bold) as well as Japanese human isolates reported previously (Asakura et al., 2006; Hagiwara et al., 2006; Hatanaka et al., 2003; Komiya et al., 2010; Noguchi et al., 2009; Nureki et al., 2007) were analysed by PFGE as described in Methods. The phylogenetic tree was generated by the UPGMA method.

of the $C$. diphtheriae NCTC 13129 genome sequence strain. We also determined the complete tox gene sequences of $C$. diphtheriae culture collection strains ATCC 11049 (GenBank accession no. AB602356), ATCC 11051 (GenBank accession no. AB602357) and RIMD 0343044 (GenBank accession no. AB602358) and of a clinical isolate, CD1994-1 (GenBank accession no. AB602359). These tox sequences differed from that of the $C$. diphtheriae genome sequence but only by $0-2$ bases. The deduced amino acid sequences of all five $C$. diphtheriae strains were identical.

\section{Antibiotic sensitivity}

None of the strains were resistant to benzylpenicillin, ampicillin, cefazolin, cefotiam, cefotaxime, cefaclor, cefditoren, flomoxef, imipenem, meropenem, erythromycin, minocycline, vancomycin or sulfamethoxazole-trimethoprim. The CLDM MIC was $\geqslant 2 \mu \mathrm{g} \mathrm{ml}^{-1}$ in all strains, judged as resistant or intermediate. In addition, the LVFX
MIC was $2 \mu \mathrm{g} \mathrm{ml}^{-1}$ in six isolates and $\leqslant 0.25 \mu \mathrm{g} \mathrm{ml}^{-1}$ in the remaining 39 isolates.

\section{DISCUSSION}

In this study, 42 toxigenic and three non-toxigenic $C$. ulcerans isolates were obtained from dogs in the custody of the Osaka Prefectural Government from November 2007 to December 2008. The isolates were divided into five groups and three subgroups according to the period of isolation, as shown in Table 2. Further analysis showed that these groupings correlated with PFGE types and tox gene sequence types.

Group 1 isolates were obtained from four (50\%) of eight dogs with the same guardian, suggesting a group infection by $C$. ulcerans in an asymptomatic state. The guardian was looking after these dogs in a pasture in the riverbed. The source of infection was unclear; however, many types of

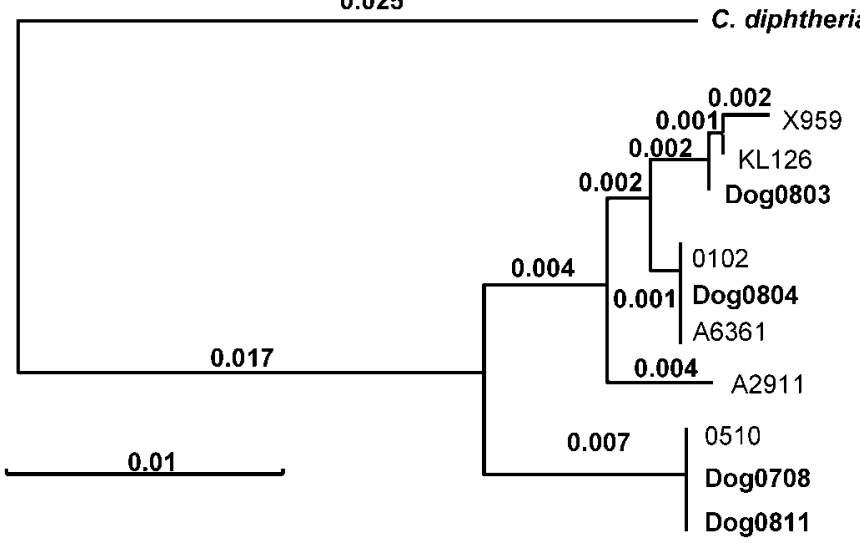

Fig. 3. Phylogenetic analysis based on nucleotide sequences of the tox genes. The tox gene sequences for the C. ulcerans isolates obtained in the present study were determined and compared to the previously published tox gene sequences of $C$. ulcerans strains isolated from humans and the $C$. diphtheriae strain NCTC 13129. A dendrogram was generated by the neighbour-joining method (Saitou \& Nei, 1987). 
Table 3. Nucleotide sequence (roman) and deduced amino acid (italic) similarity (\%) of tox genes

\begin{tabular}{|c|c|c|c|c|c|c|c|}
\hline tox type or strain & tox0811 & A2911 & tox0804 & tox0803 & KL126 & X959 & $\begin{array}{l}\text { C. diphtheriae } \\
\text { NCTC } 13129\end{array}$ \\
\hline A2911 & 98.5 & & 99.5 & 99.3 & 99.1 & 98.6 & 94.8 \\
\hline tox0804 & 98.6 & 99.4 & & 99.8 & 99.6 & 99.1 & 95.0 \\
\hline tox0803 & 98.5 & 99.1 & 99.7 & & 99.8 & 99.3 & 94.8 \\
\hline X959 & 98.4 & 98.9 & 99.5 & 99.8 & 99.8 & & 94.5 \\
\hline NCTC 13129 & 95.1 & 95.0 & 95.1 & 95.2 & 95.1 & 95.1 & \\
\hline
\end{tabular}

animals inhabit the fields, and they possibly played a role in C. ulcerans transmission.

Group 2 organisms were isolated from $27(28 \%)$ of 98 dogs examined in 2 months from April 4 to June 3. These dogs came, not from a specific area, but from a wide range of locations in the Osaka Prefecture. Dogs residing in the Osaka Prefectural Dog Management Office were usually euthanized after being boarded for about 1 week. Because dogs at the Dog Management Office were not kept in isolation, they had many opportunities for physical contact. Therefore, C. ulcerans was possibly transmitted from one dog to another during the boarding period in the facility. This hypothesis is supported by our results indicating identical PFGE and tox gene sequence types for all isolates belonging to group 2. Furthermore, after group 2 dogs were cleared from the boarding facility after sampling on June 3,C. ulcerans was not detected in the 45 dogs tested during the subsequent sampling period.

Group 3 was composed of a cluster of isolates obtained subsequent to group 2. This group (six isolates) had the same PFGE type (A2) as group 2 and group 4-1 isolates; however, the LVFX MIC in group 3 isolates was higher than that for group 2 and group 4-1 isolates. We therefore concluded that group 3 isolates originated from different sources to those for the isolates in groups 2 and 4-1. As with group 2, each of the six dogs in group 3 was transported to the facility from geographically distant regions of Osaka Prefecture. Thus, these C. ulcerans isolates might have been transmitted from one dog to another in the facility, rather than acquired from dogs residing at their original locations.

The next cluster of isolates was designated group 4, isolated from six dogs in September and October. Although transmission is assumed to have occurred within the facility, four strains were toxigenic while the other three were not. Two distinct PFGE patterns for the three isolates (A2 for nos 40 and 42, and B for no. 41) characterized non-toxigenic strains, indicating their different origins. In contrast, isolate nos 39 and 40 (one toxigenic and the other non-toxigenic, respectively) were obtained from a single dog (Table 2). Apart from their toxigenicity, these isolates were indistinguishable, sharing the same API code, PFGE type and antibiotic resistance pattern. The tox gene of C. ulcerans is known to be carried by bacteriophages (Seto et al., 2008). Toxigenicity in these two isolates might be the result of tox gene acquisition through infection by and lysogenization of a bacteriophage. Alternatively, the non-toxigenic isolate might have been the result of loss of tox-bearing bacteriophage from the toxigenic organism. Further analysis will support this hypothesis if isogenicity between these isolates is confirmed.

Additionally, another isolate was obtained in November and was classified in group 5. This isolate exhibited an independent PFGE pattern from those of the other isolates (Table 2, Figs 1 and 2).

Although C. ulcerans can cause mouth ulcers (Lartigue et al., 2005) and bronchopneumonia (Sykes et al., 2010), all C. ulcerans-positive dogs were asymptomatic, regardless of the toxigenicity of the isolate. Our studies suggest that although weakly virulent, C. ulcerans is readily transmitted among dogs. The lack of $C$. ulcerans isolation in winter suggests that fewer contacts occurred between the animals during cold weather due to reduced activity.

In our previous study in 2009 (Katsukawa et al., 2009), the rate of incidence $(1 / 65)$ was considerably lower than the incidence $(45 / 583)$ in the present study. The apparent discrepancy might be due to the difference in the sampling population: in the previous study, the population examined was supposed to be less than $10 \%$ of the total number of dogs in the custody, and the results obtained there might not have reflected the actual carrier rate.

Among the 45 C. ulcerans isolates, 42 were toxigenic. The phylogenetic tree and sequence comparisons are shown in Fig. 3 and Table 3, respectively. All tox genes of C. ulcerans formed a monocluster distinct from that of the tox gene of C. diphtheriae, which is in agreement with the results of previous studies (Seto et al., 2008; Sing et al., 2003, 2005). It is thus unlikely that the tox genes detected in the $C$. ulcerans isolates obtained in this study could have been transmitted from C. diphtheriae. In Brazil, a non-toxigenic C. ulcerans strain has also been isolated from a dog kept in an animal shelter (Dias et al., 2010). In this case, transmission between dogs was not observed.

C. diphtheriae-selective medium containing potassium tellurite has been routinely used for years (Efstratiou \& 
George, 1999), but its selective ability for other corynebacteria has not been fully evaluated. Using the broth (Mueller-Hinton broth) microdilution method, we found that the MIC of potassium tellurite for C. ulcerans and $C$. diphtheriae was $0.03 \%$ and $0.125 \%$, respectively. The cell density of C. ulcerans at $0.03 \%$ potassium tellurite was fourfold less than that of $C$. diphtheriae at $0.125 \%$ (data not shown). Tinsdale agar medium, which is frequently used to selectively isolate C. diphtheriae (Tinsdale, 1947), has been assumed to support the growth of C. ulcerans as well. This medium contains approximately $0.03 \%$ potassium tellurite; however, its concentration differs according to the manufacturer. Therefore, C. ulcerans strains could show relatively poor growth on Tinsdale agar. As reported previously, a medium was developed for the efficient culture of C. ulcerans (Katsukawa et al., 2009). By adding activated charcoal and blood to Mueller-Hinton broth, the MIC for C. ulcerans increased to $0.125 \%$. We therefore used charcoal-tellurite blood agar, which contains activated charcoal $(0.05 \%)$, potassium tellurite $(0.03 \%)$ and sheep blood $(10 \%)$; the composition was based on heart infusion agar, which is used to detect C. ulcerans. This agar medium enabled us to isolate $C$. ulcerans from 44 of 583 dogs in the present study. All $C$. ulcerans isolates were obtained from the charcoal-tellurite blood agar, but only three from the blood agar. These data show that charcoal-tellurite blood agar is suitable for the selective isolation of $C$. ulcerans from samples that contained small numbers of $C$. ulcerans and many other normal flora bacteria from dogs' throats.

The strains isolated in this study were all sensitive to $\beta$ lactam or macrolide antibiotics, which are often used to treat diphtheria (Bonnet \& Begg, 1999). In contrast, all strains were judged as intermediate resistant or resistant to CLDM, and six strains were intermediately resistant to LVFX. Although reports of erythromycin- and CLDMresistant (Tiwari et al., 2008), erythromycin-resistant (Schuhegger et al., 2009; Tiwari et al., 2008) and CLDMintermediate resistant strains (Sykes et al., 2010) have been published, resistance to quinolones has not been reported until now. A report of failure in curing the bacterium by enrofloxacin, another fluoroquinolone antibiotic, has been described (Sykes et al., 2010). Since the CLSI does not publish a standard LVFX MIC for Corynebacterium species, six strains with an LVFX MIC of $2 \mu \mathrm{g} \mathrm{ml}^{-1}$ could not be classified as susceptible. The increased resistance of some C. ulcerans strains to LVFX indicates that care should be taken in administering quinolones to treat infections.

Corynebacterial infections in dogs are difficult to cure: amoxicillin ( $2 \mathrm{~g}$ daily for 15 days), enrofloxacin (5 mg $\mathrm{kg}^{-1}$ ) and doxycycline $\left(5.8 \mathrm{mg} \mathrm{kg}^{-1}\right.$ orally every $12 \mathrm{~h}$ for 10 weeks) treatments have been reported to be unsuccessful (Hogg et al., 2009; Lartigue et al., 2005; Sykes et al., 2010). Mechanisms to account for the ineffectiveness of these antibiotics remain to be determined. The drugs may be less effective due to metabolic factors in the host, an inability to access the bacteria, or inefficient transport into the pathogen. Alternatively, the host may be repeatedly infected.

Since there would be great resistance to euthanizing companion animals to eliminate a bacterial reservoir, controlling infections by an antibiotic-independent way is of utmost importance. Development of an effective vaccine to prevent animals from infection may provide an alternative to antibiotic treatment. Existing diphtheria toxoid vaccines are considered to be effective in protecting humans from toxaemic diseases caused by $C$. ulcerans (De Zoysa et al., 2005; Tiwari et al., 2008). However, the toxoid vaccine is not considered to prevent animals and humans from infection because its nature is chemically or physically detoxified diphtheria toxin. Vaccines effective against infection, possibly by utilizing bacterial components essential for colonization, are thus desired, especially because non-toxigenic C. ulcerans can be converted to toxigenic by bacteriophages. Additional studies need to be conducted to identify candidate antigens for a suitable animal vaccine.

\section{ACKNOWLEDGEMENTS}

This work was supported in part by Health and Labour Sciences Research Grants (Research on Emerging and Re-emerging Infectious Diseases and Research on Pharmaceutical and Medical Safety, H19Shinkou-Ippan-009).

\section{REFERENCES}

Asakura, S., Katayama, N., Hara, Y., Sunami, K., Ohgami, T., Takeda, Y., Mimura, S., Yamatori, I., Ohkusu, K. \& other authors (2006). A case of cervico-lymphadenitis due to Corynebacterium ulcerans, 2005 Okayama. Infect Agents Surveill Rep 27, 124-125 (in Japanese).

Bonnet, J. M. \& Begg, N. T. (1999). Control of diphtheria: guidance for consultants in communicable disease control. World Health Organization. Commun Dis Public Health 2, 242-249.

Bostock, A. D., Gilbert, F. R., Lewis, D. \& Smith, D. C. M. (1984). Corynebacterium ulcerans infection associated with untreated milk. J Infect 9, 286-288.

De Zoysa, A., Efstratiou, A., George, R. C., Jahkola, M., VuopioVarkila, J., Deshevoi, S., Tseneva, G. Y. \& Rikushin, Y. (1995). Molecular epidemiology of Corynebacterium diphtheriae from northwestern Russia and surrounding countries studied by using ribotyping and pulsed-field gel electrophoresis. J Clin Microbiol 33, 10801083.

De Zoysa, A., Hawkey, P. M., Engler, K., George, R., Mann, G., Reilly, W., Taylor, D. \& Efstratiou, A. (2005). Characterization of toxigenic Corynebacterium ulcerans strains isolated from humans and domestic cats in the United Kingdom. J Clin Microbiol 43, 4377-4381.

Dias, A. A. S. O., Silva, F. C., Jr, Pereira, G. A., Souza, M. C., Camello, T. C. F., Damasceno, J. A. L. D., Pacheco, L. G. C., Miyoshi, A., Azevedo, V. A. \& other authors (2010). Corynebacterium ulcerans isolated from an asymptomatic dog kept in an animal shelter in the metropolitan area of Rio de Janeiro, Brazil. Vector Borne Zoonotic Dis 10, 743-748.

Efstratiou, A. \& George, R. C. (1999). Laboratory guidelines for the diagnosis of infections caused by Corynebacterium diphtheriae and $C$. ulcerans. World Health Organization. Commun Dis Public Health 2, 250-257. 
Engler, K. H., Glushkevich, T., Mazurova, I. K., George, R. C. \& Efstratiou, A. (1997). A modified Elek test for detection of toxigenic corynebacteria in the diagnostic laboratory. J Clin Microbiol 35, 495498.

Hagiwara, N., Horike, S., Kasahara, S., Suganuma, T., Aizawa, T., Nakamura, K., Nakanishi, M., Yatsuhashi, R., Okazaki, N. \& other authors (2006). A diphtheria-like case due to Corynebacterium ulcerans, July 2006 - Kanagawa. Infect Agents Surveill Rep 27, 334-335 (in Japanese).

Hatanaka, A., Tsunoda, A., Okamoto, M., Ooe, K., Nakamura, A., Miyakoshi, M., Komiya, T. \& Takahashi, M. (2003). Corynebacterium ulcerans diphtheria in Japan. Emerg Infect Dis 9, 752-753.

Hatanaka, A., Kamata, T., Tazaki, A., Honda, K., Yamamoto, A., Komiya, T. \& Takahashi, M. (2011). A case of Corynebacterium ulcerans diphtheria - Ibaraki. Infect Agents Surveill Rep 32, 19-20 (in Japanese).

Hogg, R. A., Wessels, J., Hart, J., Efstratiou, A., De Zoysa, A., Mann, G., Allen, T. \& Pritchard, G. C. (2009). Possible zoonotic transmission of toxigenic Corynebacterium ulcerans from companion animals in a human case of fatal diphtheria. Vet Rec 165, 691-692.

Jacherts, D. (1956). [The DSS agar, a polyvalent culture medium for the differentiation of diphtherial and pseudodiphtherial bacteria] Zentralbl Bakteriol [Orig] 167, 306-309 (in German).

Katsukawa, C., Kawahara, R., Inoue, K., Ishii, A., Yamagishi, H., Kida, K., Nishino, S., Nagahama, S., Komiya, T. \& other authors (2009). Toxigenic Corynebacterium ulcerans isolated from the domestic dog for the first time in Japan. Jpn J Infect Dis 62, 171-172.

Khamis, A., Raoult, D. \& La Scola, B. (2004). rpoB gene sequencing for identification of Corynebacterium species. J Clin Microbiol 42, 3925-3931.

Komiya, T., Seto, Y., De Zoysa, A., Iwaki, M., Hatanaka, A., Tsunoda, A., Arakawa, Y., Kozaki, S. \& Takahashi, M. (2010). Two Japanese Corynebacterium ulcerans isolates from the same hospital: ribotype, toxigenicity and serum antitoxin titre. J Med Microbiol 59, 1497-1504.

Lartigue, M.-F., Monnet, X., Le Flèche, A., Grimont, P. A. D., Benet, J.-J., Durrbach, A., Fabre, M. \& Nordmann, P. (2005). Corynebacterium ulcerans in an immunocompromised patient with diphtheria and her dog. J Clin Microbiol 43, 999-1001.

Miyamura, K., Tajiri, E., Ito, A., Murata, R. \& Kono, R. (1974). Micro cell culture method for determination of diphtheria toxin and antitoxin titres using VERO cells. II. Comparison with the rabbit skin method and practical application for seroepidemiological studies. J Biol Stand 2, 203-209.

Nakao, H. \& Popovic, T. (1997). Development of a direct PCR assay for detection of the diphtheria toxin gene. J Clin Microbiol 35, 16511655.

Nakao, H., Pruckler, J. M., Mazurova, I. K., Narvskaia, O. V., Glushkevich, T., Marijevski, V. F., Kravetz, A. N., Fields, B. S., Wachsmuth, I. K. \& Popovic, T. (1996). Heterogeneity of diphtheria toxin gene, tox, and its regulatory element, $d t x R$, in Corynebacterium diphtheriae strains causing epidemic diphtheria in Russia and Ukraine. J Clin Microbiol 34, 1711-1716.
Noguchi, Y., Tsunoda, A., Ken, K., Komiya, T., Yamamoto, A. \& Takahashi, M. (2009). A case of acute pharyngitis caused by Corynebacterium ulcerans, January 2009 - Tokyo. Infect Agents Surveill Rep 30, 188-189 (in Japanese).

Nureki, S.-i., Miyazaki, E., Matsuno, O., Takenaka, R., Ando, M., Kumamoto, T., Nakano, T., Ohkusu, K. \& Ezaki, T. (2007). Corynebacterium ulcerans infection of the lung mimicking the histology of Churg-Strauss syndrome. Chest 131, 1237-1239.

Reinhardt, D. J., Lee, A. \& Popovic, T. (1998). Antitoxin-in-membrane and antitoxin-in-well assays for detection of toxigenic Corynebacterium diphtheriae. J Clin Microbiol 36, 207-210.

Saitou, N. \& Nei, M. (1987). The neighbor-joining method: a new method for reconstructing phylogenetic trees. Mol Biol Evol 4, 406425.

Schuhegger, R., Schoerner, C., Dlugaiczyk, J., Lichtenfeld, I., Trouillier, A., Zeller-Peronnet, V., Busch, U., Berger, A., Kugler, R. \& other authors (2009). Pigs as source for toxigenic Corynebacterium ulcerans. Emerg Infect Dis 15, 1314-1315.

Seto, Y., Komiya, T., Iwaki, M., Kohda, T., Mukamoto, M., Takahashi, M. \& Kozaki, S. (2008). Properties of corynephage attachment site and molecular epidemiology of Corynebacterium ulcerans isolated from humans and animals in Japan. Jpn J Infect Dis 61, 116-122.

Sing, A., Hogardt, M., Bierschenk, S. \& Heesemann, J. (2003). Detection of differences in the nucleotide and amino acid sequences of diphtheria toxin from Corynebacterium diphtheriae and Corynebacterium ulcerans causing extrapharyngeal infections. J Clin Microbiol 41, 4848-4851.

Sing, A., Bierschenk, S. \& Heesemann, J. (2005). Classical diphtheria caused by Corynebacterium ulcerans in Germany: amino acid sequence differences between diphtheria toxins from Corynebacterium diphtheriae and C. ulcerans. Clin Infect Dis 40, 325-326.

Sykes, J. E., Mapes, S. L., Lindsay, L. L., Samitz, E. \& Byrne, B. A. (2010). Corynebacterium ulcerans bronchopneumonia in a dog. J Vet Intern Med 24, 973-976.

Takahashi, M. (2009). Risk Assessment of Zoonotic Diseases by Ecological Approach, H19-Shinkou-ippan-009. Tokyo: Ministry of Health, Labour and Welfare.

Tinsdale, G. F. W. (1947). A new medium for the isolation and identification of $C$. diphtheriae based on the production of hydrogen sulphide. J Pathol Bacteriol 59, 461-466.

Tiwari, T. S., Golaz, A., Yu, D. T., Ehresmann, K. R., Jones, T. F., Hill, H. E., Cassiday, P. K., Pawloski, L. C., Moran, J. S. \& other authors (2008). Investigations of 2 cases of diphtheria-like illness due to toxigenic Corynebacterium ulcerans. Clin Infect Dis 46, 395-401.

Wellinghausen, N., Sing, A., Kern, W. V., Perner, S., Marre, R. \& Rentschler, J. (2002). A fatal case of necrotizing sinusitis due to toxigenic Corynebacterium ulcerans. Int J Med Microbiol 292, 59-63.

Yoshimura, Y., Yamamoto, A. \& Komiya, T. (2010). A case of axillary lymph node abscess caused by percutaneous infection of Corynebacterium ulcerans through scratch by a pus-discharging cat, June 2010. Infect Agents Surveill Rep 31, 331 (in Japanese). 